\title{
HICSS-53 Minitrack
}

\section{Personal Health and Wellness Management with Technologies}

\author{
Prof. Dr. Freimut Bodendorf \\ University of Erlangen- \\ Nuremberg, Germany \\ freimut.bodendorf@,fau.de
}

\author{
Prof. Nilmini Wickramasinghe \\ Swinburne University and \\ Epworth HealthCare, Australia \\ nilmini.work@gmail.com
}

\author{
Tuan Huy Ma \\ Fraunhofer Institute IIS \\ Erlangen, Germany \\ tuan-huy.ma@scs.fraunhofer.de
}

Today, empowering patients and focusing on patient centered care delivery is a critical success factor. Technology is a key enabler in this regard with mobile Apps, wearables, social media and individualized services dramatically influencing how patients and their families can manage their health and wellness. Further, they have the potential to facilitate and enhance superior healthcare delivery by clinicians and caregivers as well as assist in addressing many of the challenges currently facing healthcare delivery in all OECD countries. Moreover, these solutions foster active patient participation in their care as well as promote self-management of wellness and fitness; essential aspects in managing chronic diseases. In addition, the data collected from these solutions have the potential to enable sophisticated services for selfcare, sustainable wellness management and valuebased care to ensue.

Hence, this minitrack focuses on how such technologies and digital services might be utilized to address the challenges currently facing healthcare delivery such as escalating cost pressures, a growing aging population, an increasing prevalence of chronic diseases, and a move to a preventive care focus. Integral to these approaches is a patient-centric view in order to satisfy consumer expectations, provide high quality care, and improve wellness. This minitrack is highly interdisciplinary and brings together attendees interested in technical solutions, behavioral aspects, medical impacts, and business value of personal health and wellness management.

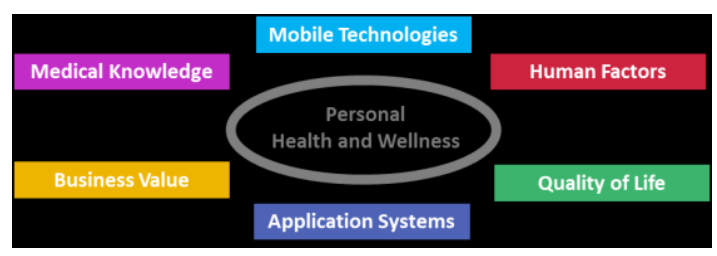

This minitrack comprises eight papers. It brings together theory and practice by presenting scientific concepts and methods as well as empirical investigations and case studies.

A first set of presentations provides a more general view and addresses information technologies, quality factors, data sharing, and privacy aspects in personal health and wellness management. Results of literature reviews, empirical studies, and framework conceptualization are introduced. The second set of presentations focuses on system design and the application of innovative methods and tools in different illustrating areas. These areas comprise wellness monitoring, stress measurement, cognitive screening, and personalized health information. Technologies applied include among others various wearable sensors, virtual reality solutions as well as natural language and text processing.

The presentations comprise reviews, research designs as well as empirical studies and particularly introduce exemplary prototypical systems. Taken together, the contributions serve to exhibit and illustrate the potentials and limits of modern technologies for personal health management and wellness.

For seven years now this minitrack has been home to a persistent and growing community of academic researchers and healthcare professionals who are interested in the possibilities of innovative technologies and IT support for individual health and wellness. We will continue to anticipate future potentials of actual trends like artificial intelligence, socio-technical environments as well as health ecosystems and platforms. 